\title{
ROLE OF ARTIFICIAL INTELLIGENCE IN DIABETES MANAGEMENT
}

\author{
Devendra Kumar Mishra ${ }^{*}{ }^{凶}$, Shubham Shukla ${ }^{2}$ \\ ${ }^{* 1}$ Amity Institute of Pharmacy, Amity University Uttar Pradesh Lucknow Campus, India \\ 2 School of Biomedical and Pharmaceutical Sciences, Babasaheb Bhimrao Ambedkar University, \\ India
}

DOI: https://doi.org/10.29121/ijetmr.v7.i7.2020.728

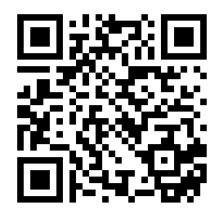

Article Citation: Devendra Kumar Mishra, and Shubham Shukla (2020). ROLE OF ARTIFICIAL INTELLIGENCE IN DIABETES MANAGEMENT. International Journal of Engineering Technologies and Management Research, 7(7), 80-88.

https://doi.org/10.29121/ijetmr.v7 i7 2020.728

Published Date: 26 July 2020

Keywords:

Diabetes Mellitus

Hyperglycemia

Machine Learning

Artificial Intelligence

Hypoglycemia

\section{ABSTRACT}

According to some of the surveys researchers also claimed that at the end of 2040 there will be marked high in the number of patients worldwide at around 600 million. The vast majority of our day by day exercises have gotten computerized. Computerized wellbeing considers the everexpanding cooperative energy between cutting edge clinical advancements, development, and computerized correspondence. DM is a condition instigated by unregulated diabetes that may prompt multi-organ disappointment in patients. Because of advances in AI and man-made brainpower which empowers the early discovery and analysis of DM through a computerized procedure which is more favorable than a manual finding. Standards of AI have been utilized to assemble calculations to help prescient models for the danger of creating diabetes or its resulting difficulties. Computer-based intelligence will present a change in perspective in diabetes care from ordinary administration systems to building focused on information-driven exactness care. As per the patient's very own need, an appropriate diabetes care plan requires various fields of experts together to make up the arrangement. So, on the off chance that it is done physically, it would limit the experience and information on these experts and devour bunches of costly clinical assets also.

\section{INTRODUCTION}

Lately, propelled clinical innovation, improved living conditions, and better general wellbeing have brought populace's future increments bit by bit. So now we are confronting a considerable developing number of constant infection patients. AI can be characterized as methods for PCs to do errands that would regularly require human insight [1]. Diabetes mellitus is an interminable, inescapable condition that is information-rich and with an assortment of possible results. In this way, diabetes is a fruitful ground for fusing simulated intelligence [2]. According to some of the surveys researchers also claimed that at the end of 2040 there will be marked high in the number of patients worldwide at around 600 million [3]. Keeping up blood glucose in an objective range (70-180 $\mathrm{mg} / \mathrm{dL}$ ) can assist with forestalling confusions identified with hyperglycemia $(>180 \mathrm{mg} / \mathrm{dL})$ and hypoglycemia $(<70$ $\mathrm{mg} / \mathrm{dL}$ ), be that as it may, this is hard to accomplish for the vast majority with T1D. Existing treatment procedures have developed throughout the most recent 100 years from one-time everyday insulin infusions, into various infusions of current long-acting and fast-acting insulin definitions, and the computerized insulin conveyance frameworks that are financially accessible today [4], [5]. Diabetes is related to different difficulties and critical horribleness and mortality [6]. It is critical to intercede to treat as well as forestall and make a convenient recognition

(C) 2020 The Author(s). This is an open access article distributed under the terms of the Creative Commons Attribution License, which permits unrestricted use, distribution, and reproduction in any medium, provided the original author and source are credited. 
Devendra Kumar Mishra, and Shubham Shukla

of diabetes. The board of diabetes is trying as one out of two grown-ups with diabetes is undiscovered yet $10 \%$ of worldwide wellbeing consumption (760billion USD) is spent on diabetes [7]. Computerized reasoning finds across the board use in four key zones in diabetes care including clinical choice, prescient populace hazard definition, robotized retinal screening, and patient self-administration apparatuses [8], [9]. The identification of DM might be done either physically by a clinical expert or by a programmed gadget. Any of these types of estimation of DM include advantages and downsides [10]. The primary preferred position of manual determination is that it needn't bother with any assistance from the machine for the DM location strategy, accordingly permitting the clinical expert to be an authority in the zone [11]. Frequently the side effects of DM in its underlying stage are low to such an extent that even an accomplished specialist can't completely distinguish them [12]. Because of advances in ML and AI, the sickness discovery and determination at an underlying stage by a computerized program is more likely and proficient than the manual DM acknowledgment technique [13]. Advantages incorporate a diminished remaining task at hand for clinical specialists and a little danger of deficiency brought about by people. For productive findings and benefits administration, choice emotionally supportive networks that are based on the PC may play out an energetic job [14]. The current article investigates how AI, a noticeable part of artificial intelligence, might be set to change diabetes care.

\section{DIABETES MELLITUS}

The term diabetes mellitus delineates a metabolic issue of various etiology portrayed by consistent hyperglycemia with agitating impacts of sugar, fat, and protein assimilation coming about due to blemishes in insulin discharge, insulin movement, or both [15]. Diabetes Mellitus (DM) is an issue that incorporates how the body changes over food into vitality [16]. The effects of diabetes mellitus consolidate a long bad effect on the various organs. Diabetes mellitus may give different appearances, for instance, thirst, polyuria, clouding of vision, and weight decrease [15]. In DM once one eats up food; the body changes over it into sugar named glucose and moves it to the circulatory framework. The pancreas produces insulin, which is a hormone that will by and large trade glucose from the blood to the cells that utilization it for essentialness [17]. Normally signs are not genuine or may be missing, and along these lines hyperglycemia of sufficient degree to cause fanatical and utilitarian changes may be accessible for a long time before the investigation is made [15]. If you have DM and don't search for drugs, the body doesn't make insulin as it does. Especially glucose suffers in the body, an effect, for the most part, called high glucose [18]. The varieties from the standard of starch, fat, and protein assimilation are a result of the lacking movement of insulin on target tissues coming about as a result of brutality or nonattendance of insulin [15]. DM creates in different manners, contingent upon the source [18]. The drawn-out effects of diabetes mellitus consolidate dynamic progression of the specific complexities of retinopathy with anticipated visual weakness, nephropathy that may incite renal disillusionment, or possibly neuropathy with the risk of foot ulcers, evacuation, Charcot joints, and features of autonomic and including sexual [15].

\subsection{TYPES OF DIABETES MELLITUS}

- Type 1 diabetes, also known as insulin-subordinate diabetes mellitus, happens on the grounds that the individual's invulnerable framework crushes the pancreatic beta cells. Subsequently, the pancreas delivers next to zero insulin. Type 1 diabetes for the most part creates in individuals more youthful than age 20 and it endures all through life. When manifestations of type 1 diabetes emerge, $80-90 \%$ of the islet beta cells have been crushed.

- Type 2 diabetes, also known as non-insulin-subordinate diabetes mellitus, is considerably more typical than type 1 , speaking to over $90 \%$ of all things considered. Type 2 diabetes frequently happens in corpulent individuals who are over age 35 . Be that as it may, the quantity of corpulent youngsters and adolescents with type 2 diabetes is expanding. Clinical side effects are mild, and the high glucose levels in the blood frequently can be constrained by diet, exercise, and weight reduction. Also, some type 2 diabetics need insulin, many have an adequate sum (or even an excess) of insulin in the blood. For these individuals, diabetes emerges not from a lack of insulin but since target cells become less delicate to it due to down guidelines of insulin receptors [19]. 


\section{ARTIFICIAL INTELLIGENCE}

Simulated intelligence has been depicted as 'a part of software engineering that means to make frameworks or strategies that examine data and permit the treatment of unpredictability in a wide scope of utilizations' [20]. Artificial intelligence has been concentrated in a few zones of medicinal services and clinical work on; including accuracy-based knowledge is ready to change medicine, populace wellbeing, and natural language handling [21]. The use of artificial intelligence to visual assignments, known as system vision, includes created huge enthusiasm inside the clinical network. In that capacity, simulated intelligence is accepted to be pertinent to visually orientated claims to fame, for example, radiology, pathology, ophthalmology, and dermatology [22]. The execution of man-made intelligence in medicine follows the two crucial ideas: virtual and physical. The virtual piece of simulated intelligence in medicine depends on the AI which acted in regard to the numerical computations that improve understanding through learning. The second sort of use of framework-based knowledge in medication wires physical articles, clinical gadgets, and sensibly robots taking an interest in the thought (care bots) [23]. The use of computer-based intelligence to diabetes is achievable and desirable for effective information dealing with and the advancement of instruments and gadgets for its administration. To give more secure innovation through man-made intelligence, it's prescribed to have safety plans, wellbeing saves, and procedural protections, with all vulnerabilities, recognized for all potentially specialized frameworks [24]. Specialized advances have presented wearable's, cell phones, and different devices that can help persistent checking and following of the patient's manifestations and infection status. Doctors and medicinal services experts ought to permit patients to pick computer-based intelligence helped care for the compelling administration of diabetes [25].

Components of the P7 idea of things to come of social insurance incorporate the accompanying components.

- Predictive: - In light of the data in the electronic wellbeing records and genomic information, we ought to have the option to decide a person's vulnerability to specific illnesses.

- Personalized: - The customize medication will include fitting/redoing treatment to each and every person.

- Preventive: - Rather than rewarding an infection when it causes an individual, AI, and choice expository instruments can be utilized to create methodologies to forestall the beginning of ailment.

- Precise: - When information and data are assembled, and afterward choice systematic instruments can be utilized to correctly decide the reason for infection and to suggest fitting helpful activities.

- Pervasive: - The medicinal services ought to be given whenever, anyplace, and in any area.

- Protective: - Appropriate wellbeing measures ought to be taken to guarantee that the secrecy of every single patient data is looked after.

- Participatory: - The patient ought to effectively partake in the finding and the treatment of their ailment [26].

\section{STRATEGY FOR DIABETES CARE}

A few computer-based intelligence-based procedures have been applied in diabetes care. It will help to tackle diabetes and provide the information and track of their health. There are different ways and methods by which it can be maintained.

\subsection{IN DIABETES}

Diabetes care is portrayed by the relationship between the person with diabetes, the human administrations capable, and the social protection system, which ties them. Man-made intelligence can improve care from every one of the three perspectives.

\section{The Individual with Diabetes}

Variability in blood glucose levels, related to lifestyle factors, for instance, exercise and stress, speak to a basic test to the self-organization of diabetes. This may be overpowered by smart estimations that become acquainted with the impact of these segments on an individual's glucose processing. For example, an 'Advanced Bolus 
Devendra Kumar Mishra, and Shubham Shukla

Calculator', constrained by case-based reasoning, will make altered bolus proposition once it acclimates to a person's physiology with repeated use [27]. Scientists building up the shut circle pancreas are likewise making progress toward increasingly versatile treatment. Currently 'hybrid' frameworks, computerizing basal discharge yet requiring manual contribution around dinners, are endorsed for clinical use. Ongoing investigations utilizing versatile control calculations changing in accordance with physiological information sources, for example, sugar consumption, speak to advance towards considerably more prominent automaticity [28]; nonetheless, given that producers, for example, Medtronic has caught 125 million man long periods of insulin siphon readings, AI may encourage the up and coming age of 'insightful' versatile calculations through comprehension these huge datasets, which is a test that Medtronic is handling with the assistance of the psychological wellbeing stage IBM Watson Health [29]. AI likewise encourages versatile applications to collaborate with clients in profoundly captivating manners to help remedial consistence. A model is an application to propose fitting nourishments given an individual's present glucose readings and past glycaemic reactions.

\section{The Healthcare Professional}

AI speaks to a moving clinical worldview from unbendingly characterized administration systems to information-driven exactness medication. For instance, the look forward preliminary reasoned that weight reduction intercessions don't lessen cardiovascular hazard in overweight individuals with Type 2 diabetes; nonetheless, AI-based re-investigation distinguished a subgroup of members where weight reduction was to be sure helpful and discovered that this impact was being concealed by another subgroup where there were negative impacts [30]. Whenever converted into a clinical direction, this extra granularity would change over a one-size-fitsall suggestion into more focused on guidance, conceivably profiting the two subgroups.

\section{The Healthcare System}

With the utilization of 'system vision', there is potential to completely robotize screening programs that depend on clinical picture understanding [31,32]. For instance, in diagnosing referable diabetic retinopathy, an AI model showed authority level capability contrasted and a board of ophthalmologists [31]. There is likewise also encouraging examination in screening for foot ulcers in essential consideration [32]. This methodology may prompt fewer individuals at generally safe requiring expert referral and thusly, individuals at higher hazard profiting by shorter referral delays. AI calculations are especially skilled at recognizing in danger individuals by consolidating an immense number of feeble indicators, for example, in hereditary and metabolic investigations [33]. In the event that these techniques have more prescient force than the segment factors commonly used to foresee diabetes, they could encourage more focused on intercessions and improve the viability of diabetes counteraction programs.

\subsection{CASE-BASED REASONING (CBR) IN DIABETES}

It is a computer-based intelligence procedure to tackle new issues dependent on gaining from comparable past experiences, which is in effect widely utilized in diabetes the board [34], [35]. The 4 Diabetes supportive networks are a case of it that has been utilized in care of diabetes. The framework plans to naturally identify issues in charge for glucose of blood, propose answers for the recognized issues, and recall the viable and ineffectual answers for singular patients [34]. CBR has been utilized to upgrade and individualize insulin treatment for different supper circumstances in diabetes [35].

\subsection{ARTIFICIAL NEURAL NETWORKS IN DIABETES}

Neural systems have been made to the interface and break down divergent data and assemble customized arrangements. The neural system philosophy has discovered specific and immense applications in diabetes conclusion [37]. Shrewd calculations have been developed to examine the effect of different factors on glycemic lists [38]. 
Role of Artificial Intelligence in Diabetes Management

\subsection{OTHERS}

Different strategies like help vector relapse have been applied to diabetes care. Support vector relapse has been utilized to assemble a hypoglycemia indicator. This makes a caution for preventive intercession when patients have alarmingly low degrees of blood glucose [39].

\section{APPLICATIONS}

There is a various application involved in this AI-based treatment of the diabetes patients, which majorly includes the following terms.

\section{Home Observing and Remote Administration of Diabetes Patient}

Wearable advancements to recognize movement, pulse, and other useful or physiological factors are broadly accessible by numerous suppliers, (for example, Apple Watch ${ }^{\circledR}$, Fitbit ${ }$, Samsung Galaxy®, and Garmin ${ }^{\circledR}$, among others available wearable's). In the Wearable's portion, the quantity of clients is relied upon to add up to $65.4 \mathrm{~m}$ by 2024 in India [40]. Wearable's device helps to keep up the record of calories consumed in the patients and furthermore breaks down the admission and in the outcomes give the need for more calories or there are enough for the afternoon. The wearable for diabetes executives will no doubt be one high-sway use case for the time being. As wearable insulin estimating gadgets are as of now broadly accessible, the following stage could be the computerization of insulin organization, with insignificant human mediation [41].

\section{Robotized Retinal Screening}

Deep DL estimations have been made to robotize the assurance of diabetic retinopathy [42]. The framework insight-based screening of the retina is a feasible, precise, and particularly recognized strategy for the revelation and perception of diabetic retinopathy. Persistent satisfaction for mechanized screening is similarly high with $96 \%$ patients reported as being satisfied or content with this method [43]. Convolutional neural frameworks have been set up on obliged instructive lists to deliver sore unequivocal probability maps for hemorrhages, microaneurysms, exudates, neovascularization, and conventional appearance in the retina [44].

\section{Feature Detection and Diagnosis Support}

IDx-DR is a self-overseeing structure for the illustrative screening of patients with diabetes for diabetic retinopathy, a real condition of the retina that can incite visual lack, considering fundus photos of the eye [45]. On April 11, 2018, the IDx-DR's diabetic retinopathy area contraption got FDA [46]. IDx-DR's item contains two sorts of ML figurings: the first for picture getting ready, yielding a combined portrayal of whether the photos are of sufficient quality to be fittingly separated by the illustrative estimation and the ensuing one using multilayer convolutional neural frameworks and a multistage feature bank locator, to perceive patients encountering more than gentle diabetic retinopathy (and should thusly be implied authority) and patients who don't (and thusly should be rescreened in a year) [41]. This arrangement is self-governing, implying that it doesn't in fact require a clinician to settle on a clinical choice. Likewise, the different glucose testing machine is currently generally utilized by the patients for the observing of the sugar level, it takes the example and examines the outcome.

\section{Clinical Decision Support}

It has been seen that the intelligence-based clinical decision assist instruments with having been made to envision short-and long stretch HbA1c response after insulin beginning in patients with type 2 diabetes mellitus. These gadgets in like manner help to perceive clinical elements that can affect a patient's HbA1c response. The adaptable net regularization-set up summarized an immediate model-based regarding on standard HbA1c and evaluated glomerular filtration rate is represented to reliably predict the HbA1c response after insulin commencement. Zones under the twist (AUC) of 0.80 (95\% sureness range $0.78-0.83$ ) and 0.81 (95\% 0.79-0.84), independently, are represented short and long haul HbA1c response [47]. Computer-based intelligence has been used to develop a characteristic approach for adjusting interventions in drug adherence and envisioning the threat of hospitalization in diabetes [48]. 
Devendra Kumar Mishra, and Shubham Shukla

\section{Self- Management of Diabetes by Patient}

Self- management is the key to the treatment of diabetes. With the coming of computer-based intelligence, patients are enabled to deal with their own diabetes, create information for their own boundaries, and be their own specialists for wellbeing.

\section{Self-Treatment of Diabetes by Patient}

Computer-based intelligence permits patients with diabetes to take day by day choices for diet and action. Applications have been utilized to permit patients to evaluate the quality and calorie estimation of food consumption. Responsibility for diabetes care is improved when patients catch their very own image food and evaluate what they eat [49].

\section{Exactness Dosing in Diabetes}

Artificial intelligence will assist with reminding the patients about their medicine on schedule. Mobile-based apps have been utilized to permit patients to survey the information to set for the admission of medicine and it will consequently make a caution for the patients on said time, so the patient will not forget to take medication [50].

\section{CONCLUSION}

This paper gave a careful investigation of programmed diabetic location and analysis procedures. ML can convey viable calculations supporting professionals in their everyday work, with capacities spreading over from clinical checking through model-based accuracy dosing. While the vast majority of them will just give suggestions to be looked into and considered by doctors, others are intended to work self-sufficiently and accomplish higher precision than the doctors themselves. It empowers us to reexamine diabetes and rethink the systems for anticipation and the executives of diabetes. Currently 'hybrid' frameworks, computerizing basal discharge yet requiring manual contribution around dinners, are endorsed for clinical use. Ongoing investigations utilizing versatile control calculations changing in accordance with physiological information sources, for example, sugar consumption, speak to advance towards considerably more prominent automaticity. This will assist with acquiring a component of customized care in the administration of diabetes. Artificial intelligence will assist with reminding the patients about their medicine on schedule. Computer-based intelligence permits patients with diabetes to take day by day choices for diet and action. Patients are currently being engaged to deal with their own wellbeing and doctors can give a convenient and focused on intercession through specialized stages. This development spare time and cost since information can be gathered remotely and virtual administration is supplanting the standard visits to a center. With such changes to upgrade diabetes, the executive's additional difficulties related to the utilization of simulated intelligence, of which security and privacy remain the significant ones. These issues must be handled and settled before clinical utilization of man-made intelligence-related innovation is endorsed and accessible for the patient's advantage. Later on, assessing the capability of utilizing this framework practically speaking is worth investigating. In addition, this framework isn't constrained to help diabetes care, utilize this framework in various chronic disease cares.

\section{SOURCES OF FUNDING}

None.

\section{CONFLICT OF INTEREST}

None.

\section{ACKNOWLEDGMENT}

None. 


\section{REFERENCES}

[1] Yu KH, Beam AL, Kohane IS, Artificial intelligence in healthcare, Nat Biomed Eng, 2018;2(10):719-31.

[2] Rigla M, Garca-Saez G, Pons B, Artificial intelligence methodologies and their application to diabetes, J Diabetes Sci Technol, 2018;12(2):303-10.

[3] Yau JW, Rogers SL, Kawasaki R, Global prevalence and major risk factors of diabetic retinopathy. Diabetes Care, 2012;35:556-64.

[4] Bergenstal RM, Garg S, Weinzimer SA, Buckingham BA, Bode BW, Tamborlane WV, Kaufman FR, Safety of a Hybrid Closed-Loop Insulin Delivery System in Patients with Type 1 Diabetes, JAMA, 2016;316:1407-1408.

[5] Brown SA, Kovatchev BP, Raghinaru D, Lum JW, Buckingham BA, Kudva YC, Laffel LM, Levy CJ, Pinsker JE, Wadwa RP, Six-Month Randomized, Multicenter Trial of Closed-Loop Control in Type 1 Diabetes, New Engl J Med, 2019;381:1707-1717.

[6] Papatheodorou K, Papanas N, Banach M, Papazoglou D, Edmonds M, Complications of Diabetes 2016, J Diabetes Res, 2016;2016:6989453.

[7] International Diabetes Federation (IDF). IDF diabetes atlas, 9th edition. Brussels, Belgium: International Diabetes Federation. Available at: http://www.diabetesatlas.org, Accessed on 27th June 2020.

[8] van Gemert-Pijnen JE, Nijland N, van Limburg M, Ossebaard HC, Kelders SM, Eysenbach G, Seydel ER, A holistic framework to improve the uptake and impact of eHealth technologies, J Med Internet Res, 2011;13(4):e111.

[9] Dankwa-Mullan I, Rivo M, Sepulveda M, Park Y, Snowdon J, Rhee K, Transforming Diabetes Care Through Artificial Intelligence: The Future Is Here, Popul Health Manag, 2019;22(3):229-242.

[10] Ting DSW, Cheung CY, Lim G, Tan GSW, Quang ND, Gan A, Development and Validation of a Deep Learning System for Diabetic Retinopathy and Related Eye Diseases Using Retinal Images From Multiethnic Populations With Diabetes, JAMA, 2017;318(22):2211-23.

[11] Afzali S, Yildiz 0, An effective sample preparation method for diabetes prediction, Int Arab J Inf Technol, 2018;15(6):968-973.

[12] Theera-Umpon N, Poonkasem I, Auephanwiriyakul S, Patikulsila D, Hard exudate detection in retinal fundus images using supervised learning, Neural Computing and Applications, 2019:1-18.

[13] Zou Q, Qu K, Luo Y, Yin D, Ju Y, Tang H, Predicting diabetes mellitus with machine learning techniques, Frontiers in genetics, 2018;9:515.

[14] Alghamdi M, Al-Mallah M, Keteyian S, Brawner C, Ehrman J, Sakr S, Predicting diabetes mellitus using SMOTE and ensemble machine learning approach: The Henry Ford ExercIse Testing (FIT) project, PloS one, 2017;12(7):1-15.

[15] Alberti KGMM, Zimmet PZ, Definition, Diagnosis and Classification of Diabetes Mellitus and its Complications Part 1: Diagnosis and Classification of Diabetes Mellitus Provisional Report of a WHO Consultation, Diabetic Medicine, 1998;15:539-53.

[16] Qureshi I, Ma J, Abbas Q, Recent development on detection methods for the diagnosis of diabetic retinopathy, Symmetry, 2019;11(6):749.

[17] Natarajan S, Jain A, Krishnan R, Rogye A, Sivaprasad S, Diagnostic accuracy of community-based diabetic retinopathy screening with an offline artificial intelligence system on a smartphone, JAMA ophthalmology, 2019;137(10):1182-1188.

[18] Carracedo J, Alique M, Ramirez-Carracedo R, Bodega G, Ramirez R, Endothelial extracellular vesicles produced by senescent cells: pathophysiological role in the cardiovascular disease associated with all types of diabetes mellitus, C Vascular Pharma, 2019;17(5):447-454.

[19] Tortora GJ, Derrickson B, Principles of Anatomy \& Physiology, 13th ed, USA: John Wiley \& Sons, Inc; 2012:721.

[20] Contreras I, Vehi J, Artificial Intelligence for Diabetes Management and Decision Support: Literature Review, J Med Internet Res, 2018;20(5):e10775.

[21] Davenport T, Kalakota R, The potential for artificial intelligence in healthcare, Futur Healthc J, $2019 ; 6(2): 94$.

[22] Kulkarni S, Seneviratne N, Baig MS, Khan AHA, Artificial Intelligence in Medicine: Where Are We Now?, Acad Radiol, 2020;27(1):62-70.

[23] Mishra DK, Shukla S, A New Era of Medical by Artificial Intelligence, International Journal of Engineering Technologies and Management Research, 2020;7(6):125-30. 
Devendra Kumar Mishra, and Shubham Shukla

[24] Ellahham S, Ellahham N, Simsekler MCE, Application of Artificial Intelligence in the Health Care Safety Context: Opportunities and Challenges, Am J Med Qual, 2019:1-8.

[25] Buch V, Varughese G, Maruthappu M, Artificial intelligence in diabetes care, Diabet. Med, 2018;35:495-497.

[26] Sriram RD, Reddy SSK, Artificial Intelligence and Digital Tools: Future of Diabetes Care, Clin Geriatr Med, 2020;36(3):513-25.

[27] Pesl P, Herrero P, Reddy M, Oliver N, Johnston DG, Toumazou C, Case-based reasoning for insulin Bolus Advice: evaluation of case parameters in a six-week pilot study, J Diabetes Sci Technol, 2017;11:37-42.

[28] Dassau E, Pinsker JE, Kudva YC, Brown SA, Gondhalekar R, Dalla Man C, Twelve-week 24/7 ambulatory artificial pancreas with weekly adaptation of insulin delivery settings: effect on hemoglobin a1c and hypoglycemia, Diabetes Care, 2017;40:1719-1726.

[29] Medtronic. 'Medtronic and IBM Watson Health Partner to Develop New Ways to Tackle Diabetes'. Medtronic.com. Available at http://www.medtronic.com/us-en/about/news/ibm-diabetes.html. Accessed on 27 June 2020.

[30] Baum A, Scarpa J, Bruzelius E, Tamler R, Basu S, Faghmous J, Targeting weight loss interventions to reduce cardiovascular complications of type 2 diabetes: a machine learning-based post-hoc analysis of heterogeneous treatment effects in the Look AHEAD trial, Lancet Diabetes Endocrinol, 2017;5:808-815.

[31] Gulshan V, Peng L, Coram M, Stumpe M, Wu D, Narayanaswamy A, Development and Validation of a Deep Learning Algorithm for Detection of Diabetic Retinopathy in Retinal Fundus Photographs, JAMA, 2016;316:2402.

[32] Yap MH, Chatwin KE, Ng CC, Abbott CA, Bowling FL, Rajbhandari S, A New Mobile Application for Standardizing Diabetic Foot Images, J Diabetes Sci Technol, 2018;12:169-173.

[33] Peddinti G, Cobb J, Yengo L, Froguel P, Kravic J, Balkau B, Early metabolic markers identify potential targets for the prevention of type 2 diabetes, Diabetologia, 2017;60:1740-1750.

[34] Marling C, Wiley M, Bunescu R, Shubrook J, Schwartz F, Emerging applications for intelligent diabetes management, AI Mag, 2012;33(2):67.

[35] Schmidt R, Montani S, Bellazzi R, Portinale L, Gierl L, Cased-based reasoning for medical knowledge-based systems, Int J Med Inform, 2001;64(2):355-67.

[36] Pesl P, Herrero P, Reddy M, Oliver N, Johnston DG, Toumazou C, Georgiou P, Case-based reasoning for insulin bolus advice: evaluation of case parameters in a six-week pilot study, J Diabetes Sci Technol, 2017;11:37-42.

[37] Shankaracharya, Odedra D, Samanta S, Vidyarthi AS, Computational intelligence in early diabetes diagnosis: a review, Rev Diabet Stud, 2010;7(4):252-62.

[38] He J, Baxter SL, Xu J, Zhou X, Zhang K, The practical implementation of artificial intelligence technologies in medicine, Nat Med, 2019;25:30-36.

[39] Vapnik VN, The Nature of Statistical Learning Theory, 1ed, Berlin: Springer-Verlag; 1995.

[40] Statista (2020). Wearables in the India: statistics. https://www.statista.com/outlook/319/119/wearables/india. Accessed on 29 June 2020.

[41] Angehrn Z, Haldna L, Zandvliet AS, Gil Berglund E, Zeeuw J, Amzal B, Artificial Intelligence and Machine Learning Applied at the Point of Care, Front Pharmacol, 2020;11:759.

[42] Grzybowski A, Brona P, Lim G, Ruamviboonsuk P, Abramoff M, Ting DSW, Artificial intelligence for diabetic retinopathy screening: a review, Eye (Lond), 2020;34(3):451-60.

[43] Keel S, Lee PY, Scheetz J, Zhixi Li Z, Kotowicz MA, MacIsaac RJ, He M, Feasibility and patient acceptability of a novel artificial intelligence-based screening model for diabetic retinopathy at endocrinology outpatient services: a pilot study, Sci Rep, 2018;8:4330.

[44] Lam C, Yu C, Huang L, Rubin D, Retinal lesion detection with deep learning using image patches, Invest Ophthalmol Vis Sci, 2018;59:590-6.

[45] IDx-DR (2020). About IDx-DR, Available: https://www.eyediagnosis.co/idx-dr-eu-1 Accessed on 01 July 2020.

[46] FDA (2020). De Novo classification request for IDx-DR, Available: https://www.accessdata.fda.gov/cdrh_docs/reviews/DEN180001.pdf. Accessed on 01 July 2020.

[47] Nagaraj SB, Sidorenkov G, van Boven JFM, Denig P, Predicting short- and long-term glycated haemoglobin response after insulin initiation in patients with type 2 diabetes mellitus using machine-learning algorithms, Diabetes Obes Metab, 2019;21(12):2704-11. 
[48] Lo-Ciganic WH, Donohue JM, Thorpe JM, Using machine learning to examine medication adherence thresholds and risk of hospitalization, Med Care, 2015;53:720-8.

[49] Frøisland DH, Arsand E, Integrating visual dietary documentation in mobile-phone-based self-management application for adolescents with type 1 diabetes, J Diabetes Sci Technol, 2015;9(3):541-8.

[50] Shah VN, Garg SK, Managing diabetes in the digital age, Clin Diabetes Endocrinol, 2015;1:16. 